\title{
Continued progress in understanding the molecular genetics of malignant hyperthermia
}

\author{
Henry Rosenberg, MD
}

Received: 1 March 2011 / Accepted: 17 March 2011/Published online: 1 April 2011

(C) Canadian Anesthesiologists' Society 2011

A little over 50 years ago, Denborough and Lovell described the syndrome known today as malignant hyperthermia (MH). ${ }^{1}$ Little was known about this disorder at the time other than it carried a high fatality rate and was characterized by explosive hyperthermia in an otherwise apparently healthy individual undergoing general anesthesia. The unravelling of the principal features of the syndrome involved a combination of astute clinical observations, a fortuitous association with a similar syndrome in pigs, and assiduous laboratory investigations involving contributions from many countries. Seminal contributions to the understanding of $\mathrm{MH}$ were made by individuals associated with the University of Toronto, particularly Drs. Beverly Britt, Werner Kalow, Roderick Gordon, David MacLennan, and their many coworkers.

In this issue of the Journal, Dr. MacLennan et al. continue to expand on earlier work in $\mathrm{MH}$, particularly the molecular genetics of $\mathrm{MH}$ and the allied myopathy, Central Core Disease. $^{2}$ Although the inheritance pattern of $\mathrm{MH}$ in humans was known by the 1980s, a turning point in the $\mathrm{MH}$ story occurred in 1990 when Dr. MacLennan's team identified the mutation in the ryanodine receptor gene causal for $\mathrm{MH}$ in the swine model of $\mathrm{MH} .{ }^{3}$ However, the molecular genetics of $\mathrm{MH}$ in humans has proven to be far more complex and obscure. ${ }^{4}$ This is due, in part, to the inherent complexity of the $\mathrm{MH}$ syndrome; to a variety of presentations that may be mimicked by several clinical situations; to a lack of typical phenotypic signs in the absence of anesthesia; to an association with several genes; and potentially, to scores of DNA variants that may lead to

H. Rosenberg, MD ( $₫)$

Department of Medical Education and Clinical Research,

Saint Barnabas Medical Center, Livingston, NJ, USA

e-mail: hrosenberg@sbhcs.com the pathophysiologic abnormality in $\mathrm{MH}$, namely, calcium dysregulation in the skeletal muscle cell.

Since MH may become manifest without warning and may progress rapidly to life-threatening hypercarbia, muscle breakdown, acidosis, and hyperthermia, much effort has been expended in attempting to identify those at risk for the syndrome. The mainstay diagnostic test for $\mathrm{MH}$ is the muscle biopsy contracture test first described by Drs. Britt and Kalow in the early 1970s. ${ }^{5}$ The test entails exposure of biopsied skeletal muscle to calcium-releasing agents, such as halothane, caffeine, ryanodine, and /or chloro-m-cresol. Since that time, the contracture test has served to clarify the inheritance of $\mathrm{MH}$, the association with Central Core Disease, and multiple phenotypic presentations of the syndrome. Clearly, it is not applicable either to screening or to rapid identification of susceptibility. Furthermore, as with most laboratory tests, sensitivity and specificity are not $100 \%$. Nevertheless, the test has served as the standard against which other tests are judged. One of the main current goals of research related to $\mathrm{MH}$ is the development of a minimally invasive, highly accurate diagnostic test for MH. Molecular genetics holds the promise of meeting that goal. In fact, clinical genetic testing for $\mathrm{MH}$ susceptibility is already available in many countries at the present time, but it requires careful patient selection because of the limited sensitivity of genetic testing. The road to the goal line of a sensitive and specific genetic test has proven to be replete with obstacles, just as it has been for many other inherited disorders. For instance, although the ryanodine receptor gene (RYR-1) and the ryanodine receptor or calcium release channel in the sarcoplasmic reticulum are most often associated with the syndrome, other calcium regulatory proteins, such as the dihydropyridine receptor and, more recently, calsequestrin, lead to intracellular calcium dysregulation and clinical $\mathrm{MH}$ 
as well. Secondly, the large size of the RYR-1 gene makes it extremely time-consuming to sequence the entire gene.

In addition, since not all DNA changes lead to a significant functional change in a protein, characterization of the more than 300 mutations in the RYR-1 gene in relation to calcium regulation require a functional assay. This is a laborious and detailed problem that involves either incorporating the altered gene into a cell system and analyzing calcium flux in the presence of calcium release agents or developing a "knockin" animal model that incorporates the mutation into its genome. That has been done for 30 ryanodine mutations. The effort to distinguish causal mutations from those that are not pathologic continues in several labs around the world and is one of the objectives of the study by MacLennan et al. ${ }^{2}$ In order to select mutations most likely to disrupt normal cell function, a variety of bioinformatics tools have been developed to assess those more likely to represent a change in a protein that will significantly disrupt calcium flux. Those more likely to be pathologic (as shown in Table 2 of the paper) may then be selected for more rigorous functional characterization. By enlarging the panel of causal mutations, the percent of $\mathrm{MH}$ patients who can be identified by genetic testing will be increased from the current $30 \%$ to $40 \%$ to hopefully closer to $100 \%$. It must be remembered, though, that perhaps as many as 30\% of MH cases are not related to the RYR-1 gene.

Population studies, such as the current one, provide insights into the prevalence of different mutations as well as demonstrate the likely ethnic derivation of the population at risk (genetic epidemiology). Identification of common mutations associated with $\mathrm{MH}$ will enhance the cost effectiveness of genetic testing. The current study shows that the identification of causal mutations is $42 \%$ in a highly select MH-susceptible population.

Due to the large size of the RYR-1 gene, it is not surprising that some patients harbour more than one allelic mutation, or a mutation and one or more polymorphisms, or even two causal mutations. Consequently, in a family where some members are $\mathrm{MH}$-susceptible despite one branch of the family harbouring a known causal mutation, failure to find that mutation in other family members does not rule out MH susceptibility (family C-27, Fig. 2). In addition, there is a low level but not zero discordance between a negative contracture test result and a causal mutation. The explanations for the discordance are numerous, but none is clearly proven. The cause may be problems with the contracture test, other genes or even chemicals that silence a gene, or simple laboratory error.

The authors of the current study chose a population most likely to harbour genetic changes on the basis of being MH positive by contracture testing, by the clinical grading scale, and /or by evidence of hyperCKemia. Therefore, it is not surprising that more than $80 \%$ of the patients had at least one RYR-1 mutation and about $42 \%$ harboured a causal mutation. Even so, this represents only about $20 \%$ of the MH families registered in the MH Investigational Unit's database. Including others with less well-characterized MH events may skew the numbers somewhat. Full characterization of the prevalence and significance of RYR-1 mutations will require genetic studies of MH-negative individuals as well as those many individuals who are not as well-characterized as being $\mathrm{MH}$-susceptible.

Given all the caveats, does genetic testing for $\mathrm{MH}$ detection have a place and a role? Most certainly. Investigations in many countries have demonstrated that those with positive CHCT or IVCT (CHCT is the North American version of the contracture test and IVCT is the European version) results are more likely to demonstrate one or more mutations. When such a mutation is identified in a family, other family members with that mutation may also be considered as MH-susceptible and forego biopsy testing. Furthermore, when a death occurs under circumstances that raise the suspicion of $\mathrm{MH}$, genetic testing may be extremely useful in clarifying the MH status of the descendent and his/her family. In addition, in children or others unable to undergo biopsy testing, genetic testing may also be helpful. Under those circumstances, it is important to review the clinical material carefully since genetic testing will not be productive unless there is a high clinical suspicion of the syndrome.

Studies such as this one pave the way for screening, if not routine screening then certainly targeted population screening, to determine propensity for $\mathrm{MH}$.

Malignant hyperthermia syndrome is a prototypical pharmacogenetic syndrome. It was first identified as such in the 1960s, and over the years, the syndrome continues to provide investigators with the means to understand the relation between drugs, genes, protein structure, and protein function. Until recently, studies of pharmacodynamics and pharmacokinetics were the tools available to predict drug disposition and action. However, these approaches were often incomplete. Pharmacogenomics holds the promise of a more sophisticated and detailed understanding of drug action. In my opinion, studies such as the present one and others concerning $\mathrm{MH}$ will be extremely valuable in understanding how genetics relates to clinical manifestations of diseases and drug action.

\section{Les progrès continuent dans la compréhension de la génétique moléculaire de l'hyperthermie maligne}

Il y a un peu plus de 50 ans, Denborough et Lovell décrivaient le syndrome que nous connaissons aujourd'hui sous le nom d'hyperthermie maligne (HM). ${ }^{1}$ À l'époque, 
très peu de choses étaient connues à propos de ce trouble, hormis le fait qu'il était accompagné d'un taux élevé de décès et qu'il se caractérisait par une hyperthermie explosive chez une personne autrement en bonne santé subissant une anesthésie générale. La combinaison d'observations cliniques astucieuses, d'une association fortuite de ce syndrome à un syndrome semblable chez le porc et de recherches de laboratoire assidues avec le concours de plusieurs pays, a permis de dévoiler les principales caractéristiques du syndrome d'hyperthermie maligne. Des contributions déterminantes à la compréhension de l'HM ont été apportées par des personnes associées à l'Université de Toronto, et tout particulièrement par les Drs Beverly Britt, Werner Kalow, Roderick Gordon, David MacLennan et leurs nombreux collègues.

Dans ce numéro du Journal, Dr MacLennan et coll. continuent de développer des travaux précédemment réalisés sur l'HM, particulièrement sur la génétique moléculaire de l'HM et la myopathie qui y est associée, la myopathie congénitale à axe central. ${ }^{2}$ Bien que le rôle de l'hérédité de l'HM chez l'humain soit connu depuis les années 1980, un tournant dans l'histoire de l'HM est survenu en 1990 lorsque l'équipe du Dr MacLennan a identifié une mutation des gènes récepteurs de la ryanodine provoquant l'HM dans un modèle porcin d'HM. ${ }^{3}$ Cependant, la génétique moléculaire de l'HM chez l'humain s'est avérée bien plus complexe et plus obscure. ${ }^{4}$ Ceci est dû, au moins en partie, à la complexité inhérente du syndrome d'HM, à la variété des manifestations du syndrome, qui peuvent ressembler à plusieurs situations cliniques, à l'absence de signes phénotypiques typiques en l'absence d'anesthésie, à une association à plusieurs gènes et, potentiellement, à des dizaines de variantes d'ADN qui pourraient entraîner l'anomalie physiopathologique présente dans l'HM - soit la dérégulation du calcium dans la cellule musculosquelettique.

Étant donné que l'HM peut se manifester sans signe avant-coureur et qu'elle peut progresser rapidement jusqu'à une hypercarbie potentiellement fatale, une lyse musculaire, une acidose et une hyperthermie, d'importants efforts ont été déployés afin de tenter d'identifier les personnes à risque de manifester ce syndrome. Le test diagnostique standard utilisé pour dépister une HM est la biopsie musculaire avec étude de la contractilité, d'abord décrite au début des années 1970 par les Drs Britt et Kalow. ${ }^{5}$ Dans ce test, le muscle squelettique biopsié est exposé à des agents libérant du calcium tels que l'halothane, la caféine, la ryanodine et/ou le chloro-m-crésol. Depuis, le test de contractilité a servi à clarifier l'aspect héréditaire de l'HM, son association à la myopathie congénitale à axe central et plusieurs manifestations phénotypiques de ce syndrome. Ce test n'est toutefois clairement pas réalisable pour le dépistage ou l'identification rapide d'une susceptibilité à l'HM. De plus, tout comme c'est le cas avec la plupart des tests de laboratoire, la sensibilité et la spécificité de ce test n'atteignent pas $100 \%$, mais il a néanmoins servi d'étalon or auquel comparer les autres tests. L'un des principaux objectifs actuels de la recherche liée à l'HM est la mise au point d'un test diagnostique minimalement effractif et très précis, et la génétique moléculaire promet de répondre à ces attentes. En fait, les tests génétiques cliniques permettant de détecter une susceptibilité à l'HM sont déjà disponibles actuellement dans plusieurs pays, mais ils nécessitent une sélection minutieuse des patients en raison de leur sensibilité limitée. La route jusqu'à la création d'un test génétique à la fois sensible et spécifique s'est avérée pleine d'embûches, tout comme elle l'a été dans le cas de nombreux autres troubles héréditaires. Par exemple, bien que le gène récepteur de la ryanodine (RYR-1) et le canal du récepteur de la ryanodine et de libération du calcium dans le réticulum sarcoplasmique soient le plus souvent associés au syndrome, d'autres protéines de régulation du calcium, comme le récepteur de la dihydropyridine et, plus récemment, la calséquestrine, entraînent également une dérégulation calcique intracellulaire ainsi qu'une HM clinique. Deuxièmement, la taille importante du gène RYR-1 rend son séquençage exhaustif extrêmement chronophage.

De plus, étant donné que les changements au niveau de l'ADN n'entraînent pas tous une modification fonctionnelle significative au niveau protéinique, la caractérisation des plus de 300 mutations du gène RYR-1 en relation avec la régulation calcique nécessitent un test fonctionnel. Voici un problème laborieux et minutieux, qui implique soit l'intégration du gène modifié dans un système cellulaire et l'analyse du flux calcique en présence d'agents de libération du calcium, soit la mise au point d'un modèle animal de «knock-in » qui intègre la mutation dans son génome. À ce jour, 30 mutations de la ryanodine ont été ainsi analysées. Des efforts se poursuivent dans plusieurs laboratoires du monde entier afin de différencier les mutations causales de celles qui ne sont pas pathologiques, et c'est l'un des objectifs de l'étude de MacLennan et coll. ${ }^{2}$ Afin de sélectionner les mutations les plus enclines à potentiellement perturber la fonction cellulaire normale, plusieurs outils bioinformatiques ont été mis au point qui permettent d'évaluer les mutations présentant la probabilité la plus élevée de modifier une protéine qui perturbera le flux calcique de façon significative. Les mutations les plus probablement pathologiques (voir le tableau 2 de l'article) peuvent ensuite être sélectionnées afin d'être soumises à une caractérisation fonctionnelle plus poussée. En étendant l'éventail des mutations causales, le pourcentage de patients atteints d'HM identifiables grâce à des tests génétiques augmentera des $30 \%$ à $40 \%$ actuels à un 
chiffre avoisinant - avec un peu de chance - les $100 \%$. Il convient toutefois de garder à l'esprit que jusqu'à $30 \%$ des cas d'HM pourraient ne pas être liés au gène RYR-1.

Les études de population comme celle présentée ici offrent des perspectives quant à la prévalence de diverses mutations tout en démontrant la dérivation ethnique probable de la population à risque (épidémiologie génétique). L'identification des mutations courantes associées à l'HM améliorera la rentabilité des tests génétiques. L'étude de MacLennan montre que l'identification des mutations causales est de $42 \% \mathrm{chez}$ une population rigoureusement sélectionnée de personnes susceptibles à l'HM.

En raison de la taille importante du gène RYR-1, il n'est pas surprenant que certains patients soient porteurs de plus d'une mutation allélique, ou d'une mutation et d'un ou plusieurs polymorphismes, voire de deux mutations causales. Par conséquent, dans une famille dont certains membres sont susceptibles à l'HM en dépit du fait qu'une branche de la famille porte une mutation causale connue, le fait de ne pas trouver cette mutation chez d'autres membres de la famille n'exclut pas la possibilité d'une susceptibilité à l'HM (famille C-27, figure 2). De plus, il existe une discordance, faible mais réelle, entre un résultat de test de contractilité négatif et une mutation causale. Les explications de cette discordance sont nombreuses, mais aucune n'est clairement prouvée à ce jour. Des problèmes au niveau du test de contractilité, d'autres gènes ou agents chimiques qui font taire un gène, ou une simple erreur de laboratoire peuvent en être la cause.

Les auteurs de l'étude publiée ici ont choisi une population chez laquelle il était le plus probable que des modifications génétiques soient présentes sur la base d'une HM positive selon le test de contractilité, par l'échelle de classement clinique, et/ou par la présence avérée d'hyperCKémie. Par conséquent, il n'est pas surprenant que plus de $80 \%$ des patients présentent au moins une mutation RYR-1 et qu'environ $42 \%$ soient porteurs d'une mutation causale. Mais même dans ce cas, cela ne représente qu'environ $20 \%$ des familles atteintes d'HM enregistrées dans la base de données de l'Unité de recherche sur la HM ( $M H$ Investigational Unit). L'inclusion d'autres personnes manifestant des épisodes d'HM moins bien caractérisés pourrait fausser quelque peu ces chiffres. La caractérisation complète de la prévalence et de la signification des mutations de RYR-1 nécessitera des études génétiques portant sur des personnes ne manifestant pas d'HM ainsi que sur les nombreuses personnes qui ne sont pas aussi bien caractérisées comme étant susceptibles à l'HM.

$\mathrm{Au}$ vu de tous les pièges possibles, les tests génétiques pour dépister l'HM ont-ils véritablement une place et un rôle? Très certainement. Des enquêtes réalisées dans plusieurs pays ont démontré que les personnes présentant des résultats positifs à un test de contractilité CHCT ou IVCT (le CHCT étant la version européenne du test, l'IVCT la version nord-américaine) ont plus de chances de manifester une mutation ou plus. Lorsqu'une telle mutation est identifiée dans une famille, les autres membres de la famille porteurs de cette mutation peuvent également être considérés comme susceptibles à l'HM et peuvent dès lors renoncer à la biopsie. En outre, lorsqu'un décès survient dans des circonstances éveillant une suspicion d'HM, les tests génétiques pourraient être extrêmement utiles pour déterminer le statut d'HM du descendant et de sa famille. De plus, chez les enfants et les autres personnes ne pouvant subir une biopsie, les tests génétiques pourraient également être utiles. Dans de telles circonstances, il est important de passer attentivement en revue le matériel clinique étant donné que les tests génétiques ne seront productifs que s'il existe une suspicion clinique élevée du syndrome.

Des études telles que celle-ci ouvrent la voie au dépistage de routine, ou du moins chez certaines populations ciblées, afin de déterminer leur propension à l'HM.

Le syndrome d'hyperthermie maligne est un syndrome pharmacogénétique prototypique. Ce syndrome a été identifié comme tel pour la première fois dans les années 1960 et, au fil du temps, il continue d'offrir aux chercheurs les moyens de comprendre les relations entre les médicaments, les gènes, la structure et la fonction des protéines. Jusqu'à récemment, les études de pharmacodynamie et de pharmacocinétique constituaient les outils à notre disposition pour prédire la disposition et l'action des médicaments. Ces approches étaient néanmoins souvent incomplètes. La pharmacogénomique promet de nous procurer une compréhension à la fois plus sophistiquée et plus détaillée de l'action des médicaments. Selon moi, des études telles que celle présentée ici et d'autres portant sur l'HM seront très précieuses pour comprendre la manière dont la génétique est liée aux manifestations cliniques de maladies et à l'action des médicaments.

Competing interests None declared.

\section{References}

1. Denborough MA, Lovell RR. Anaesthetic deaths in a family. Lancet 1960; 276: 45.

2. Kraeva $N$, Riazi S, Loke J, et al. Ryanodine receptor type 1 gene mutations found in the Canadian malignant hyperthermia population. Can J Anesth 2011; 58. DOI: 10.1007/s12630-011-9494-6.

3. MacLennan DH, Duff C, Zorzato F, et al. Ryanodine receptor gene is a candidate for predisposition to malignant hyperthermia. Nature 1990; 343: 559-61.

4. Rosenberg H, Sambuughin N, Dirksen R. Malignant Hyperthermia Susceptibility. GeneReviews at GeneTests: Medical Genetics 
Information Resource. Copyright, University of Washington, Seattle. Last update 2010. http://www.genetests.org. Accessed March 2011.
5. Kalow W, Britt BA, Terreau ME, Haist C. Metabolic error of muscle metabolism after recovery from malignant hyperthermia. Lancet 1970; 2: 895-8. 